\title{
Distinguishing graphs of maximum valence 3
}

\author{
Svenja Hüning* \\ Graz University of Technology \\ Graz, Austria \\ huening@tugraz . at
}

\author{
Wilfried Imrich \\ Montanuniversität Leoben \\ Leoben, Austria \\ imrich@unileoben.ac.at \\ Hannah Schreiber \\ Graz University of Technology \\ Graz, Austria \\ hannah.schreiber.k@gmail.com
}

\author{
Judith Kloas ${ }^{\dagger}$ \\ Graz University of Technology \\ Graz, Austria \\ Thomas W. Tucker ${ }^{\ddagger}$ \\ Colgate University, Hamilton \\ NY 13346, USA \\ ttucker@colgate.edu
}

Submitted: Sep 4, 2017; Accepted: Jul 19, 2019; Published: Nov 22, 2019

(C) The authors. Released under the CC BY license (International 4.0).

\begin{abstract}
The distinguishing number $D(G)$ of a graph $G$ is the smallest number of colors that is needed to color the vertices such that the only color-preserving automorphism fixes all vertices. We give a complete classification for all connected graphs $G$ of maximum valence $\Delta(G)=3$ and distinguishing number $D(G)=3$. As one of the consequences we show that all infinite connected graphs with $\Delta(G)=3$ are 2-distinguishable.
\end{abstract}

Mathematics Subject Classifications: 05C07, 05C15, 05C63

\section{Introduction}

The distinguishing number of a group $A$ acting faithfully on a set $\Omega$ is the least number of colors needed to color the elements of $\Omega$ such that any color-preserving element of $A$ fixes all elements of $\Omega$. We also call $A k$-distinguishable if the distinguishing number is at most

* Supported by Austrian Science Fund FWF W1230.

†Supported by Austrian Science Fund FWF W1230 and P24028.

${ }^{\ddagger}$ Supported by Award 317689 from the Simons Foundation. 
$k$. If $A$ is the automorphism group $\operatorname{Aut}(G)$ of a graph $G$, then the distinguishing number $D(G)$ of $G$ is the distinguishing number of the action of $A$ on the vertex set of $G$. Since its introduction for graphs by Albertson and Collins [1] more than 20 years ago, there has been an extensive literature on this topic (but see also the previous papers $[2,24]$ ) The concept has had an independent history in the theory of permutation groups $[7,26]$, unknown to graph theorists until recently [3].

The first motivation for this paper is a bound by Collins and Trenk [8] and, independently, by Klavžar, Wong and Zhu [21]. They proved that for any finite connected graph $G$ of maximum valence $\Delta(G)=d, D(G) \leqslant d+1$, with equality only if $G$ is the complete graph $K_{d+1}$, the complete bipartite graph $K_{d, d}$, or one of the cycles $C_{3}, C_{4}, C_{5}$. For infinite connected graphs the bound is the supremum of the valences, see Imrich, Klavžar and Trofimov [16]. Hence, for infinite connected graphs, $D(G) \leqslant d$, if $G$ has bounded valence $d$. If one wishes to improve this bound, it is reasonable to begin with $d=3$.

The second equally important motivation is the Infinite Motion Conjecture of Tucker [28], who conjectured that each connected, locally finite infinite graph is 2-distinguishable, if every automorphism that is not the identity moves infinitely many vertices. The conjecture is still open, although it has been shown to be true for many classes of graphs [9, 17, 27], in particular for graphs of subexponential growth [22], and thus for all graphs of polynomial growth. For a long time it was not clear whether it holds for graphs of maximal valence 3 , and whether infinite motion was really needed. This was first investigated under the additional condition of vertex transitivity [18].

It turns out that all finite or infinite connected, vertex transitive graphs of maximum valence 3 are 2-distinguishable unless they are one of four exceptional graphs. Here the result is extended to a complete classification of all finite or infinite connected graphs of maximal valence 3 that are not 2 -distinguishable.

We begin with a general observation about graphs of bounded valence. It is important to note that the distinguishing number $D(G)$ is highly dependent on the local structure of the graph $G$, as well as on its global structure. For example, suppose $G$ is a $d$-valent graph with $D(G)=1$, for $d>2$. Take an edge and subdivide it with three vertices $u, v, w$ (each has valence 2 now). Then, take a disjoint copy of $K_{d-2}$ and add edges between $u, v, w$ and each vertex in $K_{d-2}$. Now $u, v, w$ have valence $2+(d-2)=d$ and the vertices of the added $K_{d-2}$ have valence $(d-3)+3=d$. The resulting graph clearly has distinguishing number $D\left(G^{\prime}\right)=d-2$. If $d>3$, this procedure increases the distinguishing number. Thus, we would expect that any classification of graphs with valence $d>3$ would be complicated. Local structures like the added copy of $K_{d-2}$ above - we will later call them 'gadgets' play an important role in this paper.

There are infinitely many graphs with $\Delta(G)=d$ and $D(G)=d$. Let $T(n, d)$ be the tree where all vertices have valence 1 or $d$ and every vertex of valence 1 has the same distance $n$ from a root vertex $v$ (which is the unique center of the graph). Clearly, $D(T(n, d)) \geqslant d-1$, and $D(T(n, d))=d-1$ if and only if $D(T(n+1, d))=d-1$. But $T(1, d)=K_{1, d}$, so $D(T(1, d))=d$ and hence, $D(T(n, d))=d$. For $d=3$, we abbreviate $T_{n}=T(n, 3)$.

From now on we assume that all graphs have maximum valence 3, unless otherwise 
stated. We call a graph cubic if all vertices have valence 3 and subcubic if the maximum valence is 3 but there are vertices of valence 1 or 2 . We also assume that all graphs in this paper are connected, since restricting valence will not restrict the distinguishing number, if we allow enough disjoint copies of the same graph.

We say a coloring fixes a set of vertices, if any color-preserving automorphism acts as the identity on all vertices of the set. Furthermore, we will draw our rooted trees from the bottom up. This allows us to speak of up, down and cross neighbors, but we admit that it may be counter-intuitive to some readers, in particular because of the terms 'child' and 'sibling' defined in the next section.

\section{Main Theorem}

In this section we introduce three types of gadgets which will be important throughout the paper.

- Gadget of type 1: A 4-cycle uxvy with $x, y$ of valence 2.

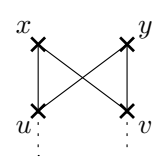

- Gadget of type 2: The same as the type 1 gadget but with an extra edge $x y$.

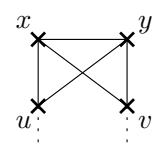

- Gadget of type 3: A hexagon uxwvzy with edges $x z$, yw (it can be viewed as $K_{2,2}$ with $u$ additionally joined to one half and $v$ to the other half, or as $K_{3,3}-u v$ ).

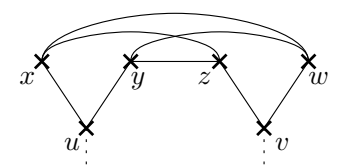

These gadgets will occur in different situations depending on the surrounding graph structure (indicated by the dotted lines above). To be more precise we first need some terminology. We call a vertex of valence 1 a leaf. If vertices $u$ and $v$ have a common neighbor, we say they are siblings or a sibling pair. Vertex $v$ is an only-child of a vertex $u$, if $v$ is the only neighbor of $u$ with valence 1 .

In every figure accompanying the definition of a gadget above there are vertices $u$ and $v$. If $u$ and $v$ have valence 3 and are siblings (resp. not siblings), call the corresponding gadget a sibling gadget (resp. non-sibling gadget). If either $u$ or $v$ has valence 2 , call it an only-child gadget. See Figure 1 for some examples. 
sibling gadget:

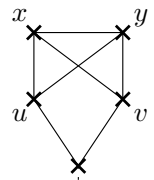

non-sibling gadget:

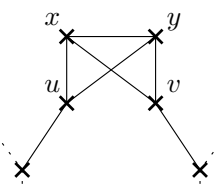

only-child gadget:

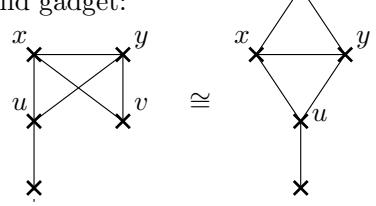

Figure 1: Examples of a sibling gadget, a non-sibling gadget and an only-child gadget.

In the first part of the paper we mostly make use of sibling gadgets to introduce some variations of $T_{n}$. The most obvious variation of $T_{n}$ is simply to join each sibling pair of leaves by an edge. Denote this graph $R_{n}^{0}$. We do this only for $n>1$ since $T_{1}$ has three sibling pairs of leaves. Adding all such edges would give $K_{4}$. Note that we can also think of $R_{n}^{0}$ as obtained from $T_{n}$ by attaching a triangle to each leaf of $T_{n-1}$. Now, we define three graphs, $R_{n}^{1}, R_{n}^{2}$ and $R_{n}^{3}$, by adding the respective sibling gadgets between each sibling pair of leaves in the tree $T_{n}$. See Figure 2 for examples.

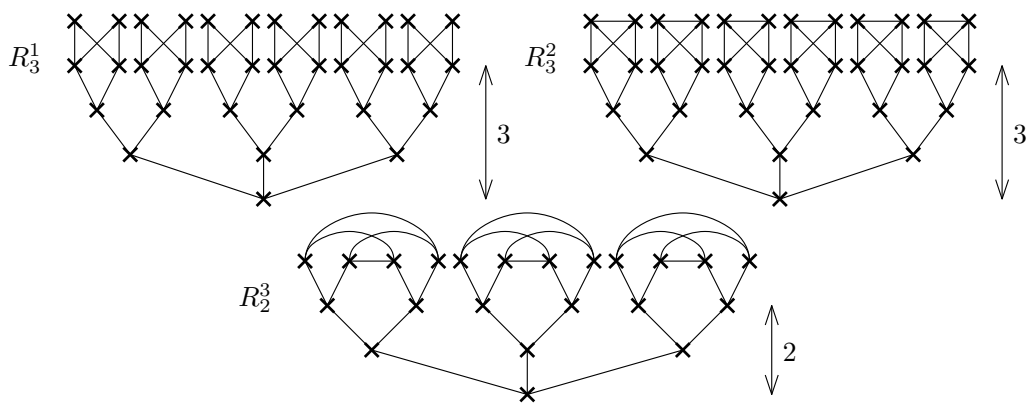

Figure 2: $R_{3}^{1}$ (top left), $R_{3}^{2}$ (top right) and $R_{2}^{3}$ (bottom).

Since $\operatorname{Aut}\left(R_{n}^{0}\right)$ acts on its vertices the same way as $\operatorname{Aut}\left(T_{n}\right)$, we have $D\left(R_{n}^{0}\right)=3$. If a distinguishing 2-coloring for one of the graphs $R_{n}^{1}, R_{n}^{2}$ or $R_{n}^{3}$ exists, then it induces a distinguishing 2-coloring of the associated $T_{n}$. Therefore, $D(G)=3$ for $G=R_{n}^{1}, R_{n}^{2}, R_{n}^{3}$.

Our classification of graphs with $\Delta(G)=3$ and $D(G)=3$ is the following:

Theorem 1 (Main Theorem). Let $G$ be a finite or infinite connected graph with $\Delta(G)=3$. Then, $D(G)=3$ if and only if $G$ is either $K_{1,3}, K_{2,3}$, the cube $Q$, the Petersen graph $P$, or a member of one of the five families $T_{n}, R_{n}^{0}, R_{n}^{1}, R_{n}^{2}, R_{n}^{3}$ for $n>1$.

We note that clearly $D\left(K_{4}\right)=D\left(K_{3,3}\right)=4$ and $D\left(K_{1,3}\right)=D\left(K_{2,3}\right)=3$. It is an exercise to verify that $D(Q)=3$ (or see [28]). It is slightly more work to show $D(P)=3$; we will sketch a proof in Section 3.

The proof that the graphs of the Main Theorem are the only graphs $G$ with maximum valence $d=3$ and $D(G)=3$ occupies most of the rest of this paper. One of the key ideas will be to show that all graphs (different from the $R_{n}^{i}$ 's) that contain a sibling, nonsibling or only-child gadget have distinguishing number 2. In Section 3, we first give some Corollaries that may shed some light on the general problem when $d>3$. In Section 4 , we 
introduce a 2-coloring which either is distinguishing or leads to restrictions on the local structure of $G$. This coloring is then used throughout the rest of the paper. In Section 5 , we show that if $G$ has leaves, then $D(G) \leqslant 2$ unless $G=T_{n}$. The goal of Section 6 is to

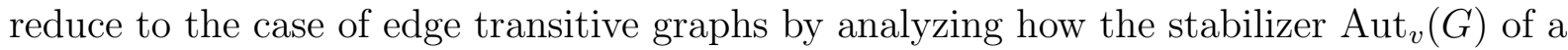
vertex $v$ acts on the neighbors of $v$. In Section 7, we show that any cubic graph $G$ with girth at least 6 has $D(G) \leqslant 2$; this proof does not use edge transitivity. This completes the proof of the Main Theorem, since the five edge transitive cubic graphs of girth less than 6 are easily analyzed. In Section 8, we pose a variety of questions. Figure 3 gives an overview of the proof strategy we use to show our Main Theorem 1.

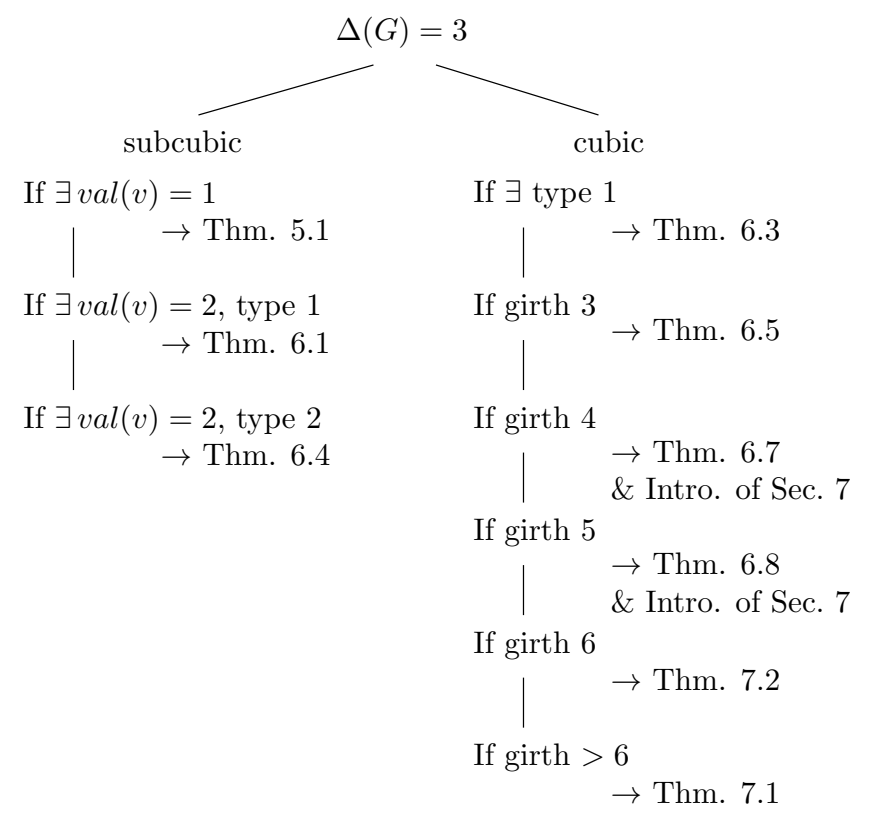

Figure 3: Overview of the case decomposition of maximal valence 3 graphs used to proof the Main Theorem 1. Note that the definition of vertex types is given at the beginning of Section 6.

\section{Corollaries for Distinguishability and Graph Structure}

We list some corollaries of the Main Theorem, mostly just observations about our list of graphs with $D(G)=3$. Each gives some insight into the relationship between distinguishing number and graph structure. Each suggests ways one might generalize the case of maximum valence 3 to graphs of higher valence. For each Corollary, $G$ is a connected graph with $\Delta(G)=3$.

Corollary 2. If $G$ is infinite, then $D(G) \leqslant 2$.

A graph $G$ is vertex transitive, if for any two vertices $u, v$ of $G$ there exists an automorphism $\alpha \in \operatorname{Aut}(G)$ such that $\alpha(u)=v$. For the definition of edge transitivity, we observe 
that each automorphism of a graph $G$ naturally acts on $E(G)$, because automorphisms preserve adjacencies. We then define $G$ to be edge transitive, if to any pair of edges $e, f$ there exists an automorphism $\beta \in \operatorname{Aut}(G)$ such the $\beta(e)=f$. For a detailed introduction to edge transitive graphs consider, for example, [14].

Corollary 3. If $G$ is vertex transitive, then $D(G) \leqslant 2$ except for $K_{3,3}, K_{4}, Q$ and $P$.

Corollary 4. If $G$ is edge transitive but not vertex transitive, then $D(G) \leqslant 2$ except for $K_{1,3}$ and $K_{2,3}$.

Corollary 5. If $G$ is 2-connected, then $D(G) \leqslant 2$, except for $K_{2,3}, K_{3,3}, K_{4}, Q$ and $P$.

Corollary 6. If $D(G)>2$, then $G$ is planar with the exception of $K_{3,3}, P$ and $R_{n}^{3}$ for $n>1$, which have minimum genus 1,1 and $3 n$, respectively.

The length of the shortest cycle in $G$ is its girth (the girth of a tree is 0).

Corollary 7. Suppose that $D(G)=3$ and $G \neq P$. Then, the girth of $G$ is at most 4. If the girth is 4, then $G=Q, R_{n}^{1}, R_{n}^{3}, K_{2,3}$. If the girth is 3, then $G=R_{n}^{0}, R_{n}^{2}$.

The motion of a group $A$ acting on a set $\Omega$ is the smallest integer $m$ such that some non-identity element of $A$ moves exactly $m$ points. The motion of a graph $G$, which we denote $m(G)$, is the motion of $\operatorname{Aut}(G)$ acting on the vertex set. The Motion Lemma $[9,25]$ states that if $m>2 \log _{2}(|A|)$, then the action has distinguishing number 2 ; the proof is elementary and short. Thus, large enough motion gives 2-distinguishability. For graphs of maximum valence 3 , large enough means 3 or more, except for $Q$ and $P$, since it is easily checked that all other graphs in our Main Theorem have motion 2. In the cases of $Q$ and $P$, it is easily verified that $m(Q)=4$ and $D(Q)=3$ (for further details see [28]), and, by Proposition 25, $m(P)=6$ and $D(P)=3$.

Corollary 8. If $m(G)>2$, then $D(G) \leqslant 2$ with the exception of $Q$ and $P$.

In fact, if $D(G)=3$ and $G$ is not $Q$ or $P$, we can isolate an automorphism of motion 2 using a 2-coloring of $G$.

Corollary 9. If $D(G)=3$ and $G$ is not $Q$ or $P$, then $G$ admits a 2-coloring that fixes all vertices except two siblings.

Proof. Clearly, such a 2-coloring exists for $K_{1,3}$ and $K_{2,3}$. All other graphs that satisfy the assumptions of the lemma have a root vertex, say $v_{0}$, corresponding to the root of $T_{n}$. The $T_{n}$ are the only graphs in the class with leaves and they all come in sibling pairs. We first construct the desired coloring for $T_{n}$.

We begin with a 2-coloring of $T_{1}$ : we color $v_{0}$ black, two of its neighbors white and one black. Clearly, this coloring fixes all vertices except for one pair of interchangeable siblings. To color $T_{n}$ we color its subgraph $T_{1}$ as before, and continue inductively by assigning different colors to any two vertices of distance $k>1$ from $v_{0}$, if they have a common neighbor of distance $k-1$ from $v_{0}$. If $k=n$, we make an exception for a single 
sibling pair of vertices whose shortest paths to $v_{0}$ contain a white neighbor of $v_{0}$. Both vertices in that pair are colored white. It is easy to see that this coloring fixes all vertices not in this pair. The same coloring works for $R_{n}^{0}$.

For $R_{n}^{1}, R_{n}^{2}$ we proceed analogously, and let the vertices which are labeled $x, y$ in the figures of the gadgets play the role of the sibling pairs in $T_{n}$. For $R_{n}^{3}$ we assign different colors to all gadget vertices $z, w$, but treat the pairs $x, y$ as before. Again, our coloring fixes all vertices except the ones in the white $(x, y)$-pair.

\section{Canonical 2-colorings rooted at a subgraph}

Let $G$ be a cubic or subcubic graph and $K$ be a vertex-induced, connected subgraph with at least two vertices. Define $S_{n}(K)$ as the set of vertices of distance $n$ from $K$; one might call it the sphere of radius $n$ around $K$. Thus, $S_{0}(K)=K$ and $S_{1}(K)$ is the set of vertices not in $K$ but adjacent to some vertex in $K$. Let $B_{n}(K)$ denote all vertices of distance at most $n$ from $K$ (the ball of radius $n$ around $K$ ). For a vertex $v$ in $S_{n}(K)$, we call its neighbors in $S_{n+1}(K), S_{n}(K), S_{n-1}(K)$, respectively, its up, cross, down neighbors. Notice that all vertices have at most two up neighbors (possibly none) and vertices not in $K$ have at least one down neighbor. We call a vertex of $K$ internal, if all its neighbors are in $K$.

The idea of the following construction is to color the spheres around $K$ inductively on $n$, after coloring all vertices of $K$. So fix an arbitrary coloring of $K$. Note that in some proofs the coloring of $K$ is important and will be mentioned explicitly. For the general definition, however, it does not matter. Our objective now is to obtain a 2-coloring of $G$ such that the only color-preserving automorphism among those fixing the vertices of $K$ is the identity. Thus, at stage $n$ we have:

Goal Assume $B_{n}(K), n \geqslant 0$, has been 2-colored so that any automorphism of $G$ fixing the vertices of $K$ and preserving the coloring of $B_{n}(K)$ is the identity on $B_{n}(K)$. Then, extend this to a 2-coloring of $S_{n+1}(K)$ that has the same property on $B_{n+1}(K)$.

The plan for extending the coloring to $S_{n+1}(K)$ is simple enough: if a vertex $v$ in $S_{n}(K)$ has a single up neighbor, color it white, and if $v$ has two up neighbors that can be switched by an automorphism of $G$ that fixes $S_{n}(K)$, color one white and one black. The problem is that the up neighbors of $v$ may have already been colored when we colored the up neighbors of a different vertex. In the following three paragraphs we will make this procedure more precise.

Assume we have colored the graph up to the sphere $S_{n}(K)$. Let $V$ be the set of vertices of $S_{n}(K)$ and $U$ be the set of vertices of $S_{n+1}(K)$. Moreover, let $H$ be the subgraph of $G$ determined by all edges between vertices of $V$ and $U$. These edges are exactly the up edges from $V$. Note that for the moment we do not care about possible cross edges in $U$ or $V$. We are interested in coloring the vertices of $U$ such that any automorphism of $G$ which fixes the vertices of $B_{n}(K)$ and preserves the coloring of $U$ also fixes the vertices of $U$. 
All vertices in $V$ have valence at most 2 in $H$. Suppose that $x \in U$ has valence 3 in $H$. If there is another vertex $y \in U$ adjacent to the same vertices in $V$ as $x$, color $x$ black and $y$ white. If there is no such vertex $y$, color $x$ white. Note that there is at most one such $y$, because all vertices in $V$ have valence at most 2 in $H$.

Now, consider the subgraph $H^{\prime}$ of $H$ obtained by removing all valence 3 vertices of $U$ in $H$, (which have already been colored). The remaining subgraph $H^{\prime}$ contains only vertices which are of valence 1 or 2 , so it is a union of paths and/or cycles such that the vertices in each component alternate between $U$ and $V$. See Figure 4 for some examples. By assumption the vertices of $V$ are fixed. Therefore, any component of $H^{\prime}$ is fixed except for two configurations. One is a 2-path, which consists of three vertices such that the middle vertex is in $V$, and the other one is a quadrilateral, see Figure 4. For all other possible components of $H^{\prime}$ there is no automorphism of $G$ fixing $B_{n}(K)$ but acting non trivially on that component. Thus, we color all vertices in $U$ which are neither in a 2-path nor in a quadrilateral white. For the remaining pairs $x, y \in U$ in a 2-path or quadrilateral, choose a coloring using black the fewest times such that any automorphism of $G$ fixing $B_{n}(K)$ also fixes these remaining pairs. Note that we know there are such colorings because we could simply color each such pair black-white. We call this a canonical 2-coloring of $G$ rooted at $K$.

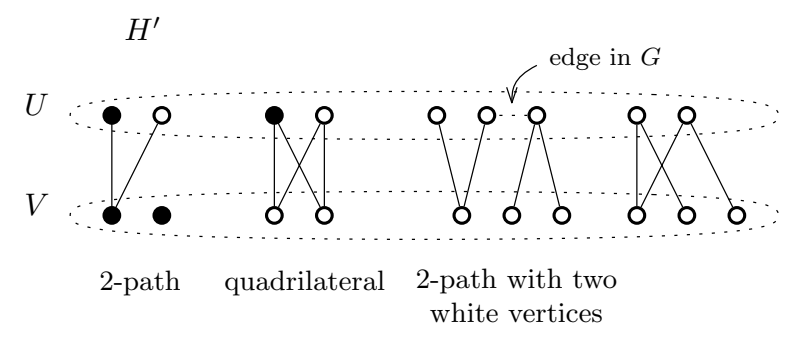

Figure 4: Possible configurations in $H^{\prime}$ used in the description of the canonical coloring.

In what follows, we use subscripts to denote which sphere a vertex is in: so $u_{n}, v_{n}, x_{n}, \ldots$ are vertices in $S_{n}(K)$. We also restrict the notion of siblings as we have defined it in Section 1 by calling two (distinct) vertices $u_{n}, v_{n}$ siblings if they have a common down neighbor.

We make the following observations about the resulting coloring:

Proposition 10 (White Up). If a vertex $v_{n}, n \geqslant 0$, has an up neighbor, it has a white up neighbor. (Note that for $n=0$ no internal vertex of $K$ has an up neighbor.)

Proposition 11 (Black-white Siblings). If a vertex $v_{n}, n \geqslant 1$, is black, it has a white sibling $u_{n}$ and there is an automorphism of $G$ interchanging $v_{n}$ and $u_{n}$ that fixes all vertices of $B_{n-1}(K)$ and all black vertices of $S_{n}(K)$.

Proof. Suppose there was no such automorphism. Then, we could color $v_{n}$ white, contradicting the minimality in the use of black. 
Proposition 12 (Black Cross). Suppose $n \geqslant 1$. If the vertices $u_{n}$ and $v_{n}$ are both black and adjacent, then there is a quadrilateral $u_{n} v_{n} x_{n} y_{n}$, where $x_{n}$ is a white sibling of $u_{n}$ and $y_{n}$ a white sibling of $v_{n}$, that is, they form a gadget of type 3 .

Proof. Since $v_{n}$ is black, by Proposition Black-white Siblings (11), it has a white sibling $y_{n}$ with an automorphism interchanging $v_{n}$ and $y_{n}$ but fixing $u_{n}$ (since it is black) forcing an edge $u_{n} y_{n}$. Similarly, since $u_{n}$ is also black there is a white vertex $x_{n}$ and edge $v_{n} x_{n}$. Since the interchange of $v_{n}$ and $y_{n}$ also leaves $x_{n}$ fixed, which is adjacent to $v_{n}$, we have $y_{n}$ adjacent to $x_{n}$.

Call a vertex of a canonical coloring kiwi, if it is black and all its neighbors are black.

Proposition 13 (All Black). The only kiwi vertices $v$ with val $(v)>1$ in a canonical coloring rooted at $K$ are in $B_{1}(K)$. Moreover, any kiwi vertex in $S_{1}(K)$ has all its neighbors in $K$.

Proof. Let $v_{n}$ be a kiwi vertex with valence $\operatorname{val}\left(v_{n}\right)>1$. By Propositions White Up (10) and Black Cross (12), all neighbors of $v_{n}$ are down neighbors. So, for $n=1$ all neighbors of $v_{n}$ are in $K$. Now, assume that $n>1$. Since $v_{n}$ is black, it has a white sibling $u_{n}$ (with the same valence) and an automorphism interchanging $u_{n}$ and $v_{n}$. Since all the neighbors of $v_{n}$ are down neighbors, $u_{n}$ has the same neighbors as $v_{n}$. Let $x_{n-1}$ be one of the common neighbors and $y_{n-1}$ be another one which exists since $\operatorname{val}\left(v_{n}\right)>1$. Because they are black and $n>1$, there is an automorphism $\varphi$ interchanging $x_{n-1}$ with a white vertex $z_{n-1}$ and fixing all other black vertices of $S_{n-1}(K)$. In particular, $\varphi$ fixes $y_{n-1}$, because it is black. Moreover, the vertices $u_{n}, v_{n}$ are the only up neighbors of $y_{n-1}$, as $n>1$. Thus, $\varphi$ can only interchange them within themselves. This forces edges from both $u_{n}$ and $v_{n}$ to the white vertex $z_{n-1}$, contradicting $v_{n}$ being kiwi and eventually having maximal valence 3 . See Figure 5.

We conclude that $n \leqslant 1$ which means that $v_{n} \in K \cup S_{1}(K)$.

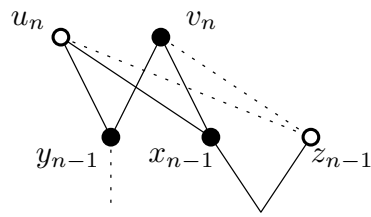

Figure 5: The vertex $v_{n}$ is a kiwi vertex. Therefore, there cannot be an edge between $v_{n}$ and $z_{n-1}$.

Proposition 14. Suppose $G$ has no vertices of valence 1 and we are given a canonical coloring of $G$ rooted at $K$ with all vertices of $K$ black. Then the only non-identity colorpreserving automorphism takes a kiwi vertex of $K$ to either another kiwi vertex of $K$ or to a kiwi vertex of $S_{1}(K)$ all of whose neighbors are in $K$. 


\section{Graphs with at least one leaf}

When dealing with infinite graphs, we will need the notion of rays: a ray is an infinite connected graph where one vertex has valence 1 and all the others have valence 2 , and a double ray is a connected infinite graph where all vertices have valence 2 . We recommend the book [11] for results on rays in infinite trees.

We first show that the only graphs $G$ with $D(G)=3$ that have a leaf are the trees $T_{n}$.

Theorem 15. If the connected subcubic graph $G$ has a leaf, then $D(G) \leqslant 2$ or $G=T_{n}$ for some $n \geqslant 1$.

Proof. The smallest two subcubic graphs with a leaf have four vertices: either $T_{1}$ or a triangle where one of the three vertices has a further neighbor. For these two graphs the theorem holds. Consider now a graph $G$ with more than four vertices. Suppose some leaf $v$ in $G$ is an only-child of $u$. The canonical coloring rooted at $K=u v$ with $u, v$ black breaks all automorphisms in $\operatorname{Aut}_{v}(G)$, since any automorphism fixing $v$ fixes $u$ as well. Since all only-children other than $v$ are colored white in a canonical coloring, there is no automorphism moving $v$, so the canonical coloring is distinguishing.

Now suppose that all leaves of $G$ come in sibling pairs and assume first that $G$ is finite. Prune all such sibling pairs in $G$. Since $\Delta(G)=3$, there is at least one leaf in the new graph. We have two cases. The new graph has again only sibling pairs. Then, prune again all sibling pairs and continue like this inductively until you get either $T_{1}$ or a graph with an only-child. If we end with $T_{1}$, we know that $G=T_{n}$ for some $n$. Otherwise we have a graph $G^{\prime}$ with an only-child, which is either subcubic or has maximal valence 2 or 1. In either case, as explained above or as mentioned in the introduction [8, 21], we can give $G^{\prime}$ a distinguishing 2-coloring and color all previously deleted sibling pairs: one sibling black and the other one white. Any automorphism $\varphi$ of $G$ takes $G^{\prime}$ to $G^{\prime}$, so if $\varphi$ is color-preserving, $\varphi$ is the identity on $G^{\prime}$. But then $\varphi$ is the identity on $G$ since all successive removed sibling pairs are colored black-white.

Now assume that $G$ is infinite. As before, if $G$ has an only-child leaf, then $D(G) \leqslant 2$. Suppose instead all leaves come in sibling pairs. We wish to prune all such pairs to form a graph $G^{\prime}$. First assume that $G$ has a cycle $C$. Now we can induct on the distance $s$ from $C$ to the closest leaf. If $s=1$, the closest leaf is an only-child, since the parent has valence 2 on the cycle $C$, so $D(G) \leqslant 2$. Assume $D(G) \leqslant 2$ for all infinite graphs with a leaf at distance $s=n$ from a cycle. Then, for $s=n+1$, either $G$ has an only-child leaf, or pruning all sibling pairs at distance $s$ gives a graph $G^{\prime}$ with $s=n$. In either case, $D(G) \leqslant 2$.

The same argument applies, if we replace cycles by double rays. Thus, it only remains to consider infinite trees without double rays (this case is also studied in [24] ). Let $G$ be such a tree. By assumption $G$ has a leaf, say $v_{0}$, and by by König's Lemma every vertex of an infinite, connected locally finite graph is the origin of a ray, see e.g. [11]. Let $R$ be a ray with origin $v_{0}$. We observe that if $v \in S_{1}(R)$, adjacent to $u \in R$, and $T_{v}$ is the component of $G-u v$ containing $v$, then $T_{v}$ must be finite: otherwise there is a ray in $T_{v}$ beginning at $v$ which combined with $u v$ and the ray in $R$ forms a double ray. 
Take a canonical coloring rooted at $K=B_{1}(R)$, with all vertices in $R$ black and all vertices in $S_{1}(R)$ white. For each $v \in S_{1}(R)$, this coloring distinguishes $T_{v}$. Let $\varphi$ be any color-preserving automorphism. Then $\varphi\left(v_{0}\right)$ cannot be in $T_{v}$ for any $v \in S_{1}(R)$, because $T_{v}$ is finite and the all black ray $\varphi(R)$ must exit $T_{v}$ through the white vertex $v$. Since all vertices in $R$ other than $v_{0}$ have valence at least two, we conclude that $\varphi\left(v_{0}\right)=v_{0}$. Since all vertices of $S_{1}(R)$ are white, $\varphi$ must fix all of $R$. Thus $\varphi$ is the identity on $G$, since every vertex in $S_{1}(R)$ has only one down neighbor in $R$. We conclude that this canonical coloring is distinguishing.

We conclude the section with the following lemma.

Lemma 16. If a connected subcubic graph $G$ has adjacent sibling vertices of valence 2 , then $D(G) \leqslant 2$ or $G=R_{n}^{0}$, for some $n \geqslant 2$. If $G$ has a sibling gadget of type 1,2 or 3 , then $D(G)=2$ or $G=R_{n}^{1}, K_{2,3}$, or $R_{n}^{2}$, or $R_{n}^{3}$, respectively, for some $n \geqslant 2$.

Proof. Suppose that $G$ has adjacent siblings of valence 2 . Thus, we know that $G$ cannot be $T_{n}$. If $G$ has a leaf, then $D(G) \leqslant 2$ by Theorem 15 . Otherwise, let $G^{\prime}$ be the graph obtained by removing all edges between sibling pairs of valence 2 . Note that $G$ cannot be just a triangle, because it is subcubic. Therefore, this procedure is possible. Then, $\operatorname{Aut}(G)$ is a subgroup of $\operatorname{Aut}\left(G^{\prime}\right)$. Thus, if $D\left(G^{\prime}\right) \leqslant 2$, we have $D(G) \leqslant 2$.

Suppose that $D\left(G^{\prime}\right)=3$. Then, $G^{\prime}=T_{n}$ by Theorem 15 , because $G^{\prime}$ has vertices of valence 1 . Since $G$ has no vertex of valence 1 , every leaf in $G^{\prime}$ comes from the removal of an edge between a sibling pair in $G$, making $G=R_{n}^{0}$. We cannot have $n=1$ here, since that would yield two adjacent triangles and thus no two adjacent siblings of valence 2. The proof for sibling gadgets of type 1,2 or 3 is the same, where $G^{\prime}$ is obtained by removing all sibling gadgets of one type, creating vertices of valence 1 , if $G$ does not only consist of a gadget. Any distinguishing 2-coloring of $G^{\prime}$ extends to one of $G$ by coloring $u, v$ and $x, y$ black-white, and for sibling gadgets of type $3, z, w$ black-white, where $v, u$,

$x, y, z$ and $w$ are the vertices as in the Figures in Section 2. Otherwise, $G=R_{n}^{1}, R_{n}^{2}, R_{n}^{3}$. If the graph only consists of a gadget, we have either $G=K_{2,3}$ or $D(G)=2$.

From this point on, we assume that none of our graphs have a vertex of valence 1. So, we do not consider graphs with leaves anymore.

\section{$6 \quad$ Vertex types}

Recall that the stabilizer $\operatorname{Aut}_{v}(G)$ of a vertex $v$ is the set of all automorphisms of $G$ that fix $v$. The general plan is to understand distinguishability of cubic or subcubic graphs by looking at the way $\operatorname{Aut}_{v}(G)$ acts on the edges incident to $v$. We note that $\operatorname{Aut}_{v}(G)$ defines a permutation group $A$ on the neighbors of $v$. If that local action is trivial, call $v$ type 1 . If the action interchanges two edges but leaves the other edge (if it exists) fixed, call it type 2. This action is described by the cyclic group $C_{2}$. We are left with the case where a vertex $v$ has valence 3 and $\operatorname{Aut}_{v}(G)$ does not fix any edge. If in this case, $\operatorname{Aut}_{v}(G)=C_{3}$, call it type 3, and otherwise, if $\operatorname{Aut}_{v}(G)$ is the symmetric group $S_{3}$, call it type 6 . Note 
that the type is the same as the order of $A$. Vertices of valence 2 can only be of type 1 or 2 . Vertices of valence 1 can only be of type 1 , but now our graphs have no vertices of valence 1. See Figure 6 for examples.

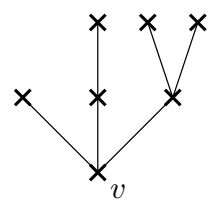

type 1

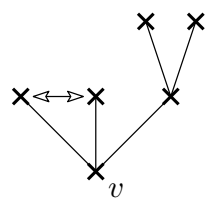

type 2

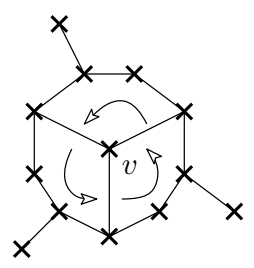

type 3

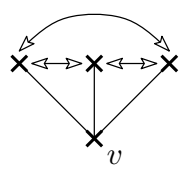

type 6

Figure 6: Examples of the different types of vertices.

Observation 1 In each of the five families $T_{n}, R_{n}^{0}, R_{n}^{1}, R_{n}^{2}, R_{n}^{3}$, the root vertex is type 6 and all other vertices of valence 3 are type 2 . For $R_{n}^{0}$, the valence 2 vertices are type 1 . For $R_{n}^{1}$, the valence 2 vertices are type 2 .

We recall that a graph is edge transitive if for any two edges $e, f$ of the graph there exists an automorphism mapping $e$ into $f$.

Observation 2 For a connected graph $G$ of maximal valence at most 3, adjacent vertices of type at least 3 imply $G$ is edge transitive and that all vertices have type at least 3 . Adjacent type 2 vertices of valence 2 imply that every vertex in $G$ is of valence 2 , and thus that $G$ is a cycle or a double ray.

\subsection{Vertices of type 1}

Theorem 17. If a connected subcubic graph $G$ without leaves has a valence 2 vertex of type 1 , then either $D(G) \leqslant 2$ or $G=R_{n}^{0}$ for some $n>1$.

Proof. Let $v$ be a valence 2 vertex of type 1 in $G$. Take a canonical coloring with $K$ the graph spanned by $v$ and its two neighbors $u$ and $w$. Color all vertices of $K$ black. By Proposition White Up (10), any non-identity, color-preserving automorphism must move $v$ to another valence 2 vertex $x$, which is either internal to $K$ (that is, all its neighbors are in $K$ ) or a kiwi vertex of $S_{1}(K)$. Note that if $v$ is fixed, then $K$ is fixed automatically, because $v$ is of type 1 .

Suppose $x$ is internal. Without loss of generality we can assume that $x=u$. Then, $u$ has valence 2 and its neighbor that is different from $v$ is inside $K$ and therefore equal to $w$. So, $K$ must be a triangle with two vertices of valence 2 and by Lemma 16 , we are done. Suppose instead that $x$ is a kiwi vertex in $S_{1}(K)$. Then, by Proposition All Black (13), the neighbors of $x$ are $u$ and $w$. But $x$ would only be black, if there is an automorphism fixing $u, w$ and interchanging $x$ with some other white vertex $z$. Then, $z$ has valence 2 as well and is adjacent to $u, w$, forcing $G=K_{2,3}$, as $v, x, z$ have valence 2 and are each adjacent to both $u$ and $w$. But then, $v$ is not a type 1 vertex. 
Corollary 18. If a connected subcubic graph $G$ contains a non-sibling or only-child gadget, then $D(G)=2$.

Proof. First, we observe, that if $G$ contains a gadget, then $D(G) \geqslant 2$. In other words, in every gadget, at least two vertices are interchangeable without coloring. Moreover, we observe that if $G$ is finite with two non-sibling adjacent vertices which are both of valence 2 , then $D(G) \leqslant 2$. The reason is that, by Observation 2 , at least one of the vertices is type 1 and, by Theorem 17 , we have $D(G) \leqslant 2$, since $R_{n}^{0}$ has no non-sibling adjacent valence 2 vertices.

First suppose that $G$ has a non-sibling gadget. Replace an appearance of a non-sibling gadget by an edge. That means, labeling a gadget as in the figures of Section 2, we delete the vertices $x$ and $y$ and connect $u$ and $v$ by an edge to create a graph $G^{\prime}$, with adjacent vertices of valence 2 that are not siblings.

If either $u$ or $v$ is of type 1 in $G^{\prime}, G^{\prime}$ finite or infinite, then, as above, $D\left(G^{\prime}\right) \leqslant 2$. One easily extends a distinguishing 2-coloring from $G^{\prime}$ to $G$, by coloring $x, y$ black-white for a gadget of type 1 or 2 and by coloring $z, w$ black-white for a type 3 gadget (with the notation as in the pictures of the gadgets in Section 2). If both $u$ and $v$ are of type 2 in $G^{\prime}$, then, by Observation $2, G^{\prime}$ is a double ray or a cycle $C_{n}$ with $n \geqslant 4$. If $G^{\prime}$ is a double ray or $n>5$, we have $D\left(G^{\prime}\right)=2$. Now assume $G^{\prime}$ is a cycle $C_{n}$ with $n=4$ or 5 . Color the endpoints of the edge replacing the gadget black-white and all other vertices of the cycle white. We can extend this coloring of $G^{\prime}$ to $G$ as explained above, which then is distinguishing in $G$.

For an only-child gadget, prune from $G$ all appearance of this only-child gadget to form a graph $G^{\prime}$. Then, $G^{\prime}$ has at least one vertex of valence 1 and by Theorem 15 , either $D\left(G^{\prime}\right) \leqslant 2$ or $G^{\prime}=T_{n}$. If $D\left(G^{\prime}\right) \leqslant 2$, we can easily extend a distinguishing 2-coloring of $G^{\prime}$ to $G$. Note that if we only remove one such only-child, we may have $D\left(G^{\prime}\right)=2$ but extending that to a distinguishing 2-coloring may require specifying a particular coloring of the other only-child gadgets.

Thus, assume that $G^{\prime}=T_{n}$. Call its center vertex $x$. Each only-child gadget has one vertex that has valence 2 in $G$ and any automorphism of $G$ can only interchange such vertices within themselves. Color one such vertex black and all the others white. This will fix one neighbor $y$ of the center $x$, so that all we need to distinguish are elements of $\operatorname{Aut}(G)$ that fix $y$ ( since $x$ is the center, $\operatorname{Aut}_{x}(G)=\operatorname{Aut}(G)$ ). This is done by coloring $y$ any color, and all other up-going siblings in $G^{\prime}=T_{n}$ black-white. Moreover, we color all up neighbors that do not have siblings white. See Figure 7 for type 2 gadgets. The picture for type 3 gadgets is similar.

Theorem 19. If a connected subcubic graph $G$ has a type 1 vertex $v$ of valence 3 , then $D(G) \leqslant 2$.

Proof. Choose a canonical 2-coloring, where $K$ is spanned by $v$ and its three neighbors $u, w, z$. Color all vertices of $K$ black. This breaks all automorphisms in $\operatorname{Aut}_{v}(G)$. Thus, the only color-preserving automorphisms left must move $v$ to a kiwi vertex $x$ of valence 3 . If no other kiwi vertex exists, then $D(G) \leqslant 2$. Otherwise, by Proposition White Up 10 , 


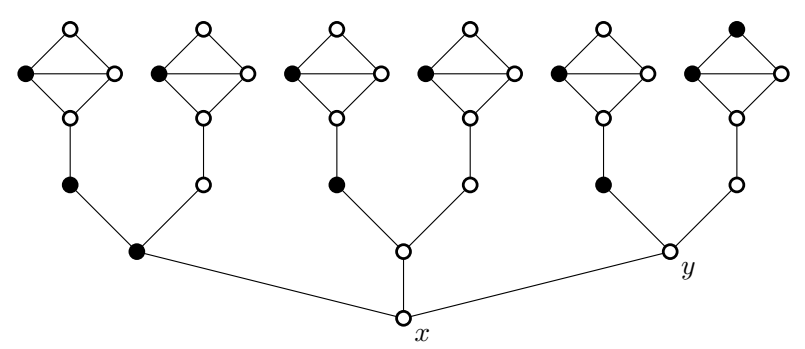

Figure 7: Example of a coloring of a graph with only-child gadgets of type 2 as described in the proof of Corollary 18.

either $x$ is internal to $K$ (that is, all it neighbors are within $K$ ) or $x \in S_{1}(K)$ and its neighbors are $u, w, z$.

Suppose that $x$ is internal to $K$, say $x=u$. Then, $u, v$ have valence 3 in $K$ and are both kiwi vertices. Moreover, by definition, the neighbors of $u$ have to be $v, w$ and $z$. If $w, z$ both have valence 3 , then $G=K_{4}$ or $K$ is a sibling or non-sibling type 2 gadget. Thus, $D(G)=2$ or $G=R_{n}^{2}$ by Corollary 18 and Lemma 16 . If $G=K_{4}$ or $R_{n}^{2}$, then $v$ is not type 1 , a contradiction. If $w, z$ both have valence 2 , then $G=K$ and again $v$ is not type 1 . If one of $w, z$ has valence 2 and the other valence 3 , we have an only-child gadget of type 2 , so $D(G)=2$ by Corollary 18 .

Suppose instead that $x \in S_{1}(K)$, which forces its down neighbors to be $u, w, z$. But $x$ would only be black if there was an automorphism fixing $K$ and interchanging $x$ with another $y \in S_{1}(K)$. This forces $G$ to be the bipartite graph $K_{3,3}$, with $v, x, y$ in one partition and $u, w, z$ in the other one. This contradicts that $v$ has type 1 .

From this point on, we assume that $G$ has no vertices of type 1.

\section{$6.2 \quad$ Type 2 vertices of valence 2}

Theorem 20. If the connected subcubic graph $G$ has no type 1 vertices, but a type 2 vertex of valence 2 , then $D(G)=2$ or $G=K_{2,3}$ or $G=R_{n}^{1}$.

Proof. There cannot be adjacent valence 2 vertices of type 2 , because then $G$ would be a cycle or double ray (both are not subcubic).

Let $G^{\prime}$ be the cubic graph obtained by smoothing over all valence 2 vertices, that is, each valence 2 vertex is removed and replaced by an edge between its neighbors. Thus, $G$ is obtained from $G^{\prime}$ by inserting a valence 2 vertex in some of its edges. If $G^{\prime}$ has multiedges, then $G$ contains either a triangle with one valence 2 and two valence 3 vertices, or a sibling or non-sibling type 1 gadget. In the latter case, $D(G)=2, G=R_{n}^{1}$, or $G=K_{2,3}$ by Lemma 16 and Corollary 18, (see Figure 8). Thus, assume that $G$ contains a triangle with two valence 3 vertices $u, w$ and one valence 2 vertex $v$. Let $K$ be the edge $v w$. Color $v$ and $w$ black and the remaining vertices with a canonical coloring rooted in $K$. Then, $u$ has to be white and any non-trivial color-preserving automorphism $\varphi$ has to move $v$ outside of $K$. Assume such a $\varphi$ exists. By Proposition 10, $\varphi(u)$ and $\varphi(w)$ cannot both be down neighbors of $\varphi(v)$ and therefore, either $\varphi(u)$ or $\varphi(w)$ is a cross neighbor of $\varphi(v)$. 
But in both cases this cross neighbor is the only sibling of $\varphi(v)$, with whom it cannot be interchanged. This means that $\varphi(v)$ cannot be black by Proposition 11. Thus, $\varphi$ does not exist and $G$ is fixed.

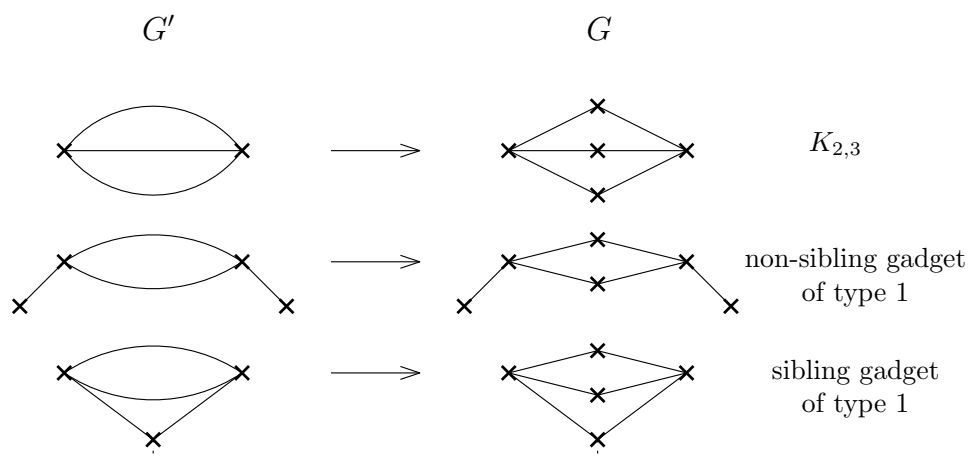

Figure 8: Left: Possibilities of multi-edges in $G^{\prime}$. Right: Origin of those multi-edges in $G$.

Assume now that $G^{\prime}$ does not have multiple edges. Note that $G^{\prime}$ is cubic. Let $w$ be one of the valence 2 vertices of $G$. Color $w$ black and color white all other valence 2 vertices in $G$. The only automorphisms preserving the coloring so far are in $\operatorname{Aut}_{w}(G)$. Let $u, v$ be the valence 3 neighbors of $w$ (that are adjacent vertices in $G^{\prime}$ ), see Figure 9. Color $v$ black and $u$ white so that they are fixed. Now the only automorphisms preserving the coloring so far are in $\operatorname{Aut}_{v}(G)$. We now proceed with a canonical coloring of the cubic graph $G^{\prime}$ rooted at $K=u v$ to distinguish $\operatorname{Aut}_{v}\left(G^{\prime}\right)$. Since $u$ and $v$ are fixed in $G$ by the coloring of the valence 2 vertex $w$, there are no color-preserving automorphisms moving $v$.

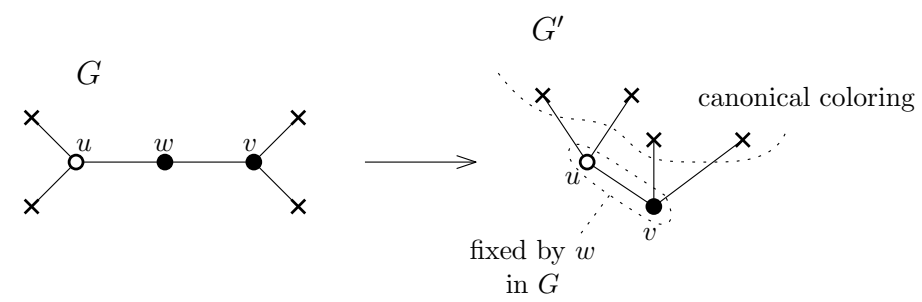

Figure 9: Coloring process in the proof of Theorem 20.

\subsection{Type 2 vertices of valence 3 , girth at most 5}

At this point we have completed the classification for subcubic graphs, since any such graph has a leaf or a valence 2 vertex of type 1 or 2 . We now consider cubic graphs with a vertex of type 2. For this proof, we use the girth of $G$, namely the length of a shortest cycle in $G$. In the next section, we show that if $G$ has girth at least 6 , then $D(G) \leqslant 2$, with no restriction on vertex types. In that section we also observe that the only edge transitive cubic graphs of girth at most 5 are $K_{4}, K_{3,3}, Q, P$ and the dodecahedron. By Observation 2, adjacent vertices of type 3 or 6 imply edge transitivity. We still assume 
that the following graphs do not contain type 1 vertices, and therefore any cycle in a graph that is not edge transitive has a type 2 vertex.

Theorem 21. Let $G$ be a connected cubic graph with girth 3 and no type 1 vertex. If $G$ contains triangles sharing an edge, then $G$ is $K_{4}, R_{n}^{2}$ or $D(G) \leqslant 2$. If $G$ contains a triangle not sharing an edge with another triangle, then $D(G) \leqslant 2$.

Proof. If two triangles of $G$ share an edge, then $G$ contains a sibling or non-sibling type 2 gadget, or $G=K_{4}$. In the first two cases cases, $G=R_{n}^{2}$ or $D(G)=2$ by Lemma 16 and Corollary 18, see Figure 10.

(1)

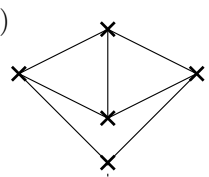

sibling gadget of type 2
(2)

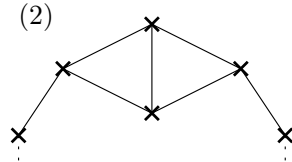

non-sibling gadget of type 2
(3)

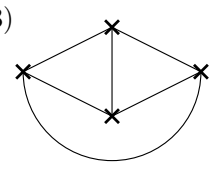

$K_{4}$

Figure 10: Two triangles sharing an edge in a cubic graph.

Assume now that there is a triangle $u v w$ in $G$ where the vertices $u^{\prime}, v^{\prime}, w^{\prime}$ adjacent to $u, v$ and $w$ respectively are distinct. Each of the vertices $u, v, w$ is of type 2: none is of type 1 and if some were of type 3 or 6 , the triangle $u v w$ would share an edge with another triangle. Thus, $\operatorname{Aut}(G)$ contains a subgroup acting transitively on the vertices $u, v, w$.

We claim that there is no automorphism taking $v$ to $v^{\prime}$. If there was, there would also be automorphisms taking $u$ to $u^{\prime}$ and $w$ to $w^{\prime}$, so each of $u^{\prime}, v^{\prime}, w^{\prime}$ lies on triangles like $u v w$ whose vertices $\operatorname{Aut}(G)$ acts on transitively. Continuing this way, we get that $G$ is vertex transitive and each vertex lies on a triangle like uvw. Let $G^{\prime}$ be the cubic graph obtained by contracting each of these triangles to a single vertex. Since each such vertex is of type 3 we have: If $G^{\prime}$ has multiple edges, then $G$ is a 3-prism in which case $D(G)=2$. Otherwise, there is a 4-coloring of $G^{\prime}$ that is distinguishing. There are also four different ways the triangles can be colored with two colors (none, one, two, or three vertices can be colored white). Thus, $G$ has a 2-coloring which fixes all triangles. But any such coloring also fixes all vertices, since any automorphism moving, say, $u$ to $v$, must also move the triangles at $u^{\prime}$ and $v^{\prime}$. Therefore, we conclude that $D(G)=2$.

Now we take a canonical coloring rooted at the graph $K$ induced by $u, v, w, u^{\prime}, v^{\prime}, w^{\prime}$, where $u^{\prime}, w$ are white and $v, v^{\prime}, u, w^{\prime}$ are black. This coloring distinguishes $\operatorname{Aut}_{v}(G)$, since any automorphism fixing $v$ also fixes all vertices in $K$ and hence all vertices of $G$. Suppose that $\varphi$ is a non-identity color-preserving automorphism. Then $\varphi(v) \neq v$. If $\varphi(v) \in K$, the only possibility is $\varphi(v)=v^{\prime}$, but we have already shown: If there is such an automorphism, then $D(G)=2$.

We conclude that $\varphi(v) \notin K$. Now, we show that there is no color-preserving automorphism $\varphi$ such that $\varphi(v) \notin K$.

Assume that such an automorphism $\varphi$ exists. Since all neighbors of $\varphi(w)$ have to be black $\varphi(w)=w_{n}, n>1$, has no up neighbor by Proposition 13. Therefore, $\varphi(u), \varphi(v)$ 
and $\varphi\left(w^{\prime}\right)$ are either also at level $n$, or are at level $n-1$. First assume that $\varphi(u)=u_{n-1}$, $\varphi(v)=v_{n-1}$ and also $\varphi\left(w^{\prime}\right)=w_{n-1}^{\prime}$. Because $w_{n-1}^{\prime}$ is black, there exists an automorphism interchanging $w_{n-1}^{\prime}$ with another vertex $z_{n-1}$ but fixing all other black vertices at level $n-1$ by Proposition 11. Thus, since $u_{n-1}$ and $v_{n-1}$ are fixed, there has to exist an extra edge from $w_{n}$ to $z_{n-1}$, contradicting the valence of $w_{n}$. See (1) in Figure 11.

Suppose instead that $\varphi\left(w^{\prime}\right)=w_{n}^{\prime}$. Now $w_{n}^{\prime}$ can be interchanged by some automorphism either with $w_{n}$ or with another vertex $z_{n}$. In the first case, since $u_{n-1}$ and $v_{n-1}$ are still fixed by Proposition 11, this forces the existence of edges from $w_{n}^{\prime}$ to $u_{n-1}$ and $v_{n-1}$. This leads to a $K_{4}$, see (3) in Figure 11. But $K_{4}$ contains triangles sharing edges which is a contradiction to our assumption. In the second case, there has to exist an extra edge from $z_{n}$ to $w_{n}$, since $u_{n-1}$ and $v_{n-1}$ are still fixed, see (2) of Figure 11 . This contradicts the valence of $w_{n}$.
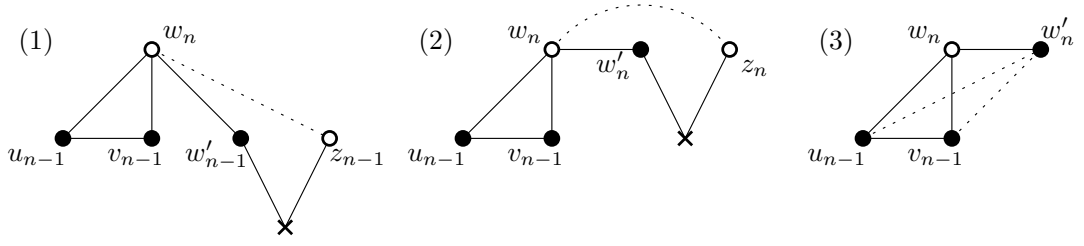

Figure 11: Possible neighborhoods of $w_{n}$ as described in the proof of Theorem 21. The dotted lines indicate necessary edges leading to a contradiction.

Consider now the situation where $\varphi(u)$ and $\varphi(v)$ are at different levels, say $\varphi(u)=u_{n-1}$ and $\varphi(v)=v_{n}$. As before, since $v_{n}$ is black, there is an automorphism interchanging $v_{n}$ with a white sibling $x_{n}$, but fixing $u_{n-1}$. If $x_{n} \neq w_{n}$, this forces an extra edge to $u_{n-1}$. If $x_{n}=w_{n}$, the automorphism also fixes $\varphi\left(w^{\prime}\right)$, forcing an edge between $v_{n}$ and $\varphi\left(w^{\prime}\right)$. This leads to adjacent triangles, whether $\varphi\left(w^{\prime}\right)=w_{n}^{\prime}$ or $\varphi\left(w^{\prime}\right)=w_{n-1}^{\prime}$, see Figure 12 .
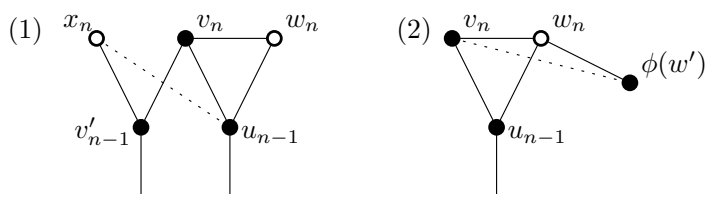

Figure 12: Possible neighborhoods of $w_{n}$ as described in the proof of Theorem 21. The dotted lines indicate necessary edges leading to a contradiction.

The remaining situation to study is when $\varphi(u)=u_{n}$ and $\varphi(v)=v_{n}$ are both on level $n$. Then, $\varphi\left(w^{\prime}\right)=w_{n-1}^{\prime}$ has to be at level $n-1$, because $\varphi(w)=w_{n}$ needs a down neighbor. Note that there is no edge between $v$ and $w^{\prime}$ and therefore none between $v_{n}$ and $w_{n-1}^{\prime}$. So, $v_{n}$ and $w_{n}$ cannot be siblings. Then, again, using the same arguments as in the precedent cases, since $v_{n}$ is black, it has a white sibling $z_{n}$ with an edge to $w_{n}$. So, we have a contradiction to the valence of $w_{n}$.

Thus, we conclude that $\varphi$ does not exist.

Corollary 22. If the connected cubic graph $G$ contains an edge-induced $K_{2,3}$, then $G=$ $K_{3,3}, R_{n}^{2}, R_{n}^{3}$ or $D(G)=2$. 
Proof. Let $u, v, w, x, y$ be vertices of an edge-induced $K_{2,3}$ where $u, v, w$ are one part and $x, y$ the other part. If there is also an edge between, say, $u$ and $v$, then we have a sibling type 2 gadget, see Figure $13(1)$. So $G=R_{n}^{2}$ or $D(G)=2$ by Lemma 16 . If $u, v$ have a common neighbor $z$, then $u, v, w, x, y, z$ form a type 3 gadget (either sibling, non-sibling or only-child, see Figure $13(2))$, so $G=K_{3,3}$ if $w$ and $z$ are also adjacent, or otherwise $R_{n}^{3}$ or $D(G)=2$ by Lemma 16 .

(1)
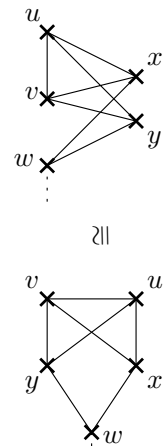

$(2)$
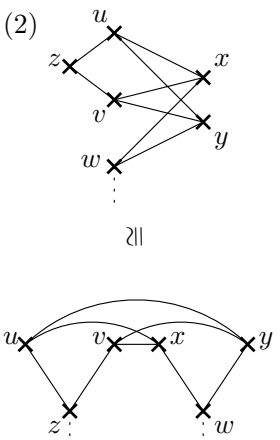

(3)

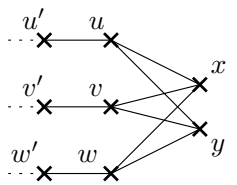

Figure 13: Possible neighborhoods of $u$ and $v$ in $K_{2,3}$. Cases (1) and (2) form two types of gadgets.

We therefore assume that the third neighbors $u^{\prime}, v^{\prime}, w^{\prime}$ of $u, v, w$, respectively, are distinct. In particular, all edge-induced copies of $K_{2,3}$ are disjoint (share no vertices). Let $G^{\prime}$ be the graph obtained by replacing each $K_{2,3}$ in the orbit ${ }^{1}$ of $u, v, w, x, y$ under $\operatorname{Aut}(G)$ by a triangle $u v w$, see Figure 14 .

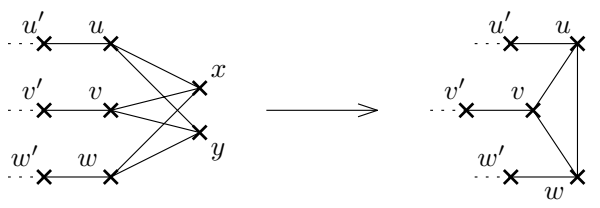

Figure 14: Substitution of $K_{2,3}$ by a triangle as in the proof of Corollary 22 .

Every automorphism of $G$ gives an automorphism of $G^{\prime}$. By Theorem 21, we have $D\left(G^{\prime}\right)=2$. We can then extend any distinguishing 2-coloring of $G^{\prime}$ to one for $G$ by coloring all pairs of vertices in the orbit of $x, y$ black-white.

Theorem 23. Let $G$ be a connected cubic graph with no type 1 vertex. If the girth of $G$ is 4 and $G$ contains a type 2 vertex, then $D(G)=2$ or $G=R_{n}^{3}$.

Proof. As mentioned at the beginning of the section, any cycle in $G$ has to contain a type 2 vertex, because $G$ is neither edge transitive nor does it contain a type 1 vertex. Let $v$ be a type 2 vertex which lies on a quadrilateral $C$. It has three neighbors $u_{1}, v_{1}$ and $w_{1}$, because $G$ is cubic. Assume without loss of generality that $v_{1}$ is fixed by $\operatorname{Aut}_{v}(G)$ and

\footnotetext{
${ }^{1}$ The orbit of a vertex $v$ consists of all images of $v$ under the action of $\operatorname{Aut}(G)$.
} 
that $u_{1}$ and $w_{1}$ can be interchanged. There are two possibilities: either the fixed edge $v v_{1}$ lies on $C$ or it does not. We will now show that in both cases the result holds.

First assume that $v v_{1}$ lies in $C$. We can then suppose without loss of generality, that $u_{1}$ also lies in $C$. Therefore, $C$ is of the form $v_{1} v u_{1} u_{2}$. But we know that $u_{1}$ and $w_{1}$ can be interchanged, so $w_{1}$ has to be in a quadrilateral $v_{1} v w_{1} w_{2}$. If $u_{2}=w_{2}$, then the vertices form a $K_{2,3}$ and by Corollary $22, D(G)=2$ or $G=R_{n}^{3}$, because the girth is 4 and $K_{3,3}$ has no type 2 vertices. Thus, assume that $u_{2} \neq w_{2}$. Let $x_{2}$ be the third neighbor of $u_{1}$. If $x_{2}=w_{1}$, we would have a triangle, which is not possible. If $x_{2}=w_{2}$, we would also need an edge between $w_{1}$ and $u_{2}$, in order to still be able to interchange $u_{1}$ and $w_{1}$. But then, the vertices form a $K_{3,3}$, contradicting $v$ being of type 2 . Thus, $x_{2} \neq w_{2}$. If there is an edge between $u_{2}$ and $w_{1}$, we have again a $K_{2,3}$ as subgraph. Therefore, the only possible configuration in this case is as shown on the left of Figure 15. The dotted edges $x_{2} w_{2}$ and $x_{2} w_{1}$ might exists or not (depending on the further structure of the neighborhoods of the vertices which have not yet valence 3 ). However, they do not interfere the remaining arguments of the proof. Let $K$ be this induced subgraph (i.e. the graph shown on the left of Figure 15). Color $v, v_{1}, u_{1}, w_{1}, u_{2}$ black and $x_{2}, w_{2}$ white. Then, color the remaining vertices in $G$ with a canonical coloring rooted in $K$. Within $K$ the vertices are clearly fixed by $\operatorname{Aut}_{v}(G)$, even if there is an edge between $x_{2}$ and $w_{1}$. Moreover, $u_{2}$ has only one up neighbor in $S_{1}(K)$, which has to be white by Proposition 10. Therefore, $v$ is the only kiwi vertex in $K$ and a non-trivial color-preserving automorphism would have to move $v$ outside of $K$. By Proposition 13 this has to be another kiwi vertex in $S_{1}(K)$. But such a vertex would have all of its neighbors in $K$, which is clearly not possible in this case. Thus, $G$ is fixed.

Now assume that the edge $v v_{1}$ does not lie in $C$. If $v v_{1}$ lies in another quadrilateral, then we can define the latter as $C$ and go back to case 1 . So, assume that $v v_{1}$ does not lie in any quadrilateral. Then, $C$ has to be of the form $u_{1} v w_{1} x_{2}$. Because $v v_{1}$ does not lie in any quadrilateral, the neighbors $v_{2}$ and $v_{2}^{\prime}$ of $v_{1}$, different from $v$, are distinct from the neighbors of $u_{1}$ and $w_{1}$. Let $u_{2}$ be the third neighbor of $u_{1}$ and $w_{2}$ be the third neighbor of $w_{1}$. If $u_{2}=w_{2}$, then $G$ contains again a $K_{2,3}$ as a subgraph and by Corollary 22 $D(G)=2$ or $G=R_{n}^{3}$. Therefore, the only possible configuration is pictured on the right of Figure 15, again with some possible additional edges, which do not interfere with the remaining proof. Let $K$ be this induced subgraph and color it as shown in the Figure. Now color the remaining vertices in $G$ with a canonical coloring rooted in $K$. As in the previous case, we see that $v$ is the only kiwi vertex in $K$ and thus, by the same arguments as above, we conclude that $G$ is fixed.

Theorem 24. Let $G$ be a connected cubic graph with no type 1 vertex. If the girth of $G$ is 5 and $G$ contains a type 2 vertex, then $D(G)=2$.

Proof. The proof is similar to the proof of the precedent Theorem 23 about graphs of girth 4. Again, we know that any cycle in $G$ has to contain a type 2 vertex. Let $v$ be a type 2 vertex which lies on a pentagon $C$. It has three neighbors $u_{1}, v_{1}$ and $w_{1}$, because $G$ is cubic. Assume without loss of generality that $v_{1}$ is fixed by $\operatorname{Aut}_{v}(G)$ and that $u_{1}$ and $w_{1}$ can be interchanged. There are two possibilities: either the fixed edge $v v_{1}$ lies on $C$ 
(1)

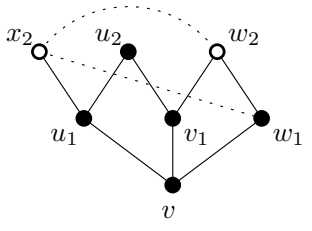

(2)

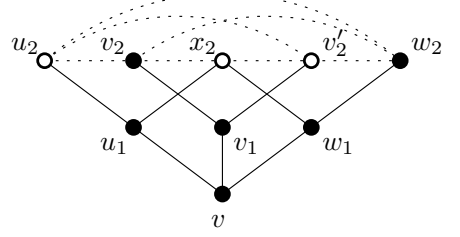

Figure 15: Subgraphs rooted in $v$ as described in the proof of Theorem 23. The dotted edges can be omitted.

or it does not. We will now show that in both cases the result holds.

First assume that $v v_{1}$ lies in $C$. We can then suppose without loss of generality, that $u_{1}$ also lies in $C$. Therefore, $C$ is of the form $v_{1} v u_{1} u_{2}^{\prime} u_{2}$. But we know that $u_{1}$ and $w_{1}$ can be interchanged, so $w_{1}$ has to lie in a pentagon $v_{1} v w_{1} w_{2}^{\prime} w_{2}$. Let $x_{2}$ be the third neighbor of $u_{1}$ and $y_{2}$ be the third neighbor of $w_{1}$. Because the girth of $G$ is 5 , the vertices $u_{1}$, $v_{1}, w_{1}$ only share one common neighbor, namely $v$. Thus, $x_{2}, y_{2}, u_{2}^{\prime}, u_{2}, w_{2}^{\prime}$ and $w_{2}$ are pairwise distinct. The configuration is shown on the left of Figure 16. Let $K$ be the subgraph induced by these vertices. Note that for simplicity we do not indicate possible cross edges here, in contrast to the previous proof. In particular, they would not interfere with the following arguments. Color $K$ as shown in Figure 16 on the left. Then, color the remaining vertices of $G$ with a canonical coloring rooted in $K$. Because the edge $v v_{1}$ is fixed by $\operatorname{Aut}_{v}(G)$, the vertex $u_{2}$ could only be interchanged with $w_{2}$. Therefore, their black-white coloring fixes the cycles which contain them and thus also fixes $u_{1}$ and $w_{1}$. So, $K$ is fixed by the coloring and in particular $G$ is fixed in $\operatorname{Aut}_{v}(G)$. It remains to verify that $v$ cannot be mapped to another kiwi vertex. In $K$, every vertex has at least one white neighbor, even $y_{2}$ : there cannot be any edge between $y_{2}$ and $w_{2}$, because it would contradict the assumption of girth 5, so, by Proposition 10, $y_{2}$ has at least one white neighbor. Therefore, any non-identity color-preserving automorphism of $G$ has to move the vertex $v$ to a kiwi vertex in $S_{1}(K)$ by Proposition 13. Assume there exists a kiwi vertex $x$ in $S_{1}(K)$. Then, its neighbors have to lie in $K$, again by Proposition 13 . Thus, they are $u_{2}^{\prime}, w_{2}$ and $y_{2}$. But the only possible up neighbor of $u_{2}^{\prime}$ has to be white by Proposition 10, and therefore $x$ cannot exist. We conclude that $G$ is fixed by the coloring.

Now we assume that $v v_{1}$ does not lie in $C$. Similar arguments as used above verify Figure 16 (2). Again, $G$ will be fixed by a canonical coloring rooted in the presented subgraph.

(1)

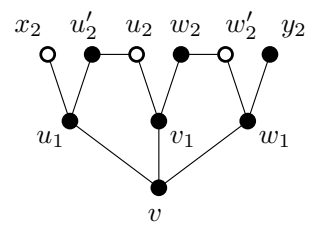

$(2)$

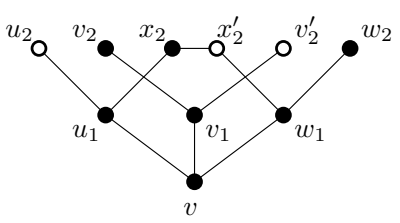

Figure 16: Subgraphs rooted in $v$ as described in the proof of Theorem 24. 


\section{Edge Transitive Graphs and Graphs of Girth at most 5}

There remain only the case where all vertices have type 3 or 6 , and the case where $G$ has a vertex of type 2 with girth at least 6 . Note that $G$ must be edge transitive in the first case by Observation 2. Our analysis falls into two parts:

(1) cubic edge transitive graphs of girth at most 5, and

(2) cubic graphs of girth at least 6 .

Theorem 21 already covers the case of maximal girth 3, but one can still easily verify directly that the only edge transitive cubic graph of girth 3 is $K_{4}$, and that $K_{3,3}$ and the cube $Q$ are the only edge transitive graphs of girth 4 . We know $D\left(K_{4}\right)=D\left(K_{3,3}\right)=4$ and $D(Q)=3$. (For a concise overview of edge transitive graphs we refer to [14].)

For girth 5, we observe that an edge transitive graph $G$ that is not vertex transitive must be bipartite: There are at most two vertex orbits for $\operatorname{Aut}(G)$, and if there are two, then adjacent vertices are in different orbits. Hence, all edge transitive graphs of odd girth are also vertex transitive. But there are only two vertex transitive cubic graphs of girth 5, the dodecahedron $H$ and the Petersen graph [13]. Note that $D(H)=2$. To find a 2-distinguishing coloring of $H$, color black a vertex and its three neighbors. Then, color also black a vertex adjacent to one of those neighbors and the remaining in white. However, $D(P)=3$, as will be shown in the following proposition.

Proposition 25. For the Petersen graph $P, D(P)=3$ and $m(P)=6$.

Proof. For the proof we take up a remark of Lehner ${ }^{2}$. He observed that $P$ is the complement of the line graph of $K_{5}$. As the line graph $L(G)$ of a graph has as its vertex set the set of edges of $G$, where any two incident edges $e, f$ are adjacent in $L(G)$, it is clear that any automorphism of $G$ induces an automorphism of $L(G)$. For small graphs $\operatorname{Aut}(L(G))$ may be larger than $\operatorname{Aut}(G)$, but if $|V(G)|>4$, then each automorphism of $L(G)$ corresponds to exactly one of $G$, see $[19,10]$.

Hence, $D\left(L\left(K_{5}\right)\right)$ is the distinguishing number of the complement of $P$, and because the automorphism group of a graph is the same (as a permutation of the set of vertices) as that of its complement, $D(P)=D\left(L\left(K_{5}\right)\right)$.

We use this to show that $D(P)>2$. Let $c$ be any 2-coloring of $P$ and let $W$ be the set of white vertices. We consider it as a set of (white) edges of $K_{5}$. Together with their end vertices they form a graph, say $G^{w}$. Because the smallest asymmetric non-trivial graph has six vertices, see [12], $G^{w}$ has at least one non-trivial automorphism. It is a non-trivial permutation of a subset of $V\left(K_{5}\right)$. We extend it to a permutation of $V\left(K_{5}\right)$ by fixing all elements $V\left(K_{5}\right) \backslash V\left(G^{w}\right)$. Because every permutation of $V\left(K_{5}\right)$ is an automorphism of $K_{5}$ we thus obtain a non-trivial automorphism of $K_{5}$. It corresponds to a non-trivial automorphism of $P$, which is color-preserving by its definition. Hence, $D(P)>2$.

To show that $D(P)=3$ it hence suffices to present a distinguishing 3-coloring. To this end we consider an arbitrary path of length 4 in $K_{5}$, color the first edge red, the other

\footnotetext{
${ }^{2}$ Private communication.
} 
two edges black, and all other edges of $K_{5}$ white. Then, the only automorphism of $K_{5}$ that preserves the edge colors is the identity, and thus $D(P)=3$.

To show that $m(P)=6$ it suffices to show that each non-trivial automorphism of $K_{5}$ moves at least six edges. Suppose $\alpha \in \operatorname{Aut}\left(K_{5}\right), \alpha(u)=v \neq u$ and $\alpha(v)=w$. If $u, v$, and $w$ are distinct, then there are six edges in $K_{5}$ with exactly one endpoint in $\{u, v, w\}$. They are all moved by $\alpha$. If $\alpha$ interchanges $u$ and $v$, then there are six edges in $K_{5}$ with exactly one endpoint in $\{u, v\}$. Again, they are all moved by $\alpha$.

Thus, we are left with (2), cubic graphs of girth at least 6 . We cannot continue with methods used for girth at most 5 , because those methods depend on using small girth to analyze the structure of $S_{2}(v)$. Instead, we return to the motivation for graph distinguishing in [1]: the Necklace Problem, that is, that $D\left(C_{n}\right)=2$ for $n \geqslant 6$.

Theorem 26. If $G$ is a connected cubic graph with girth $>6$, then $D(G) \leqslant 2$.

Proof. Let $s$ be the girth of $G$ and $C$ a cycle of length $s$. Since $s>6$, each vertex in $S_{1}(C)$ is adjacent to only one vertex in $C$. Moreover, if two vertices in $S_{1}(C)$ are adjacent, then they can be used to form a path of length three between two vertices in $C$ of distance at most $\frac{s}{2}$, contradicting the minimality of $s$. Let the vertices of $C$ be denoted by $1,2,3, \ldots, s$. Let $K$ be $C$ together with the whiskers at vertices 1 and 4 as well as $6, \ldots, s ;$ see Figure 17 for an example.

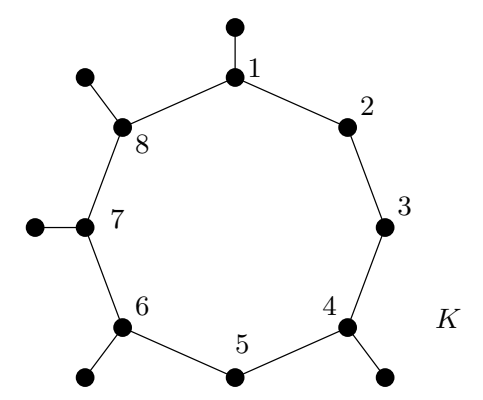

Figure 17: The set $K$ for $s=8$. The vertices 1 and 4 together with 6,7 and 8 are the kiwi vertices.

Choose a canonical 2-coloring rooted at $K$ with all vertices in $K$ colored black. By Proposition All Black (13) and Black-white Sibling (11), any kiwi vertex outside $K$ must have its three neighbors in $K$ and a white sibling with the same three neighbors. But this would create a cycle of length 4 , contradicting $s>6$. Thus, there is no kiwi vertex outside $K$. So any color-preserving automorphism $\varphi$ must leave invariant the kiwi vertices $1,4,6, \ldots, s$. The graph spanned by these vertices consists of an isolated vertex 4 and a path $6, \ldots, s, 1$. Thus, $\varphi$ fixes 4 , and either leaves the path fixed or reverses it (interchanging vertices 1,6). In the first case, $\varphi$ fixes 5 , since $s>4$, and $\varphi$ fixes 2,3 since $1, \varphi(2), \varphi(3), 4$ provides another path of length 3 between 1 and 4 , contradicting $s>6$. In the second case, $4, \varphi(5), \varphi(6)=1$ provides a path of length 2 from 4 to 1 , contradicting $s>5$. We conclude that $\varphi$ fixes all vertices of $C$ and hence, all vertices of $K$, so $\varphi$ is the identity. 
For girth $s=6$, we also have $D(G) \leqslant 2$ but the argument is slightly more complex.

Theorem 27. Let $G$ be a connected cubic graph with girth $s=6$. Then, $D(G) \leqslant 2$.

Proof. We note that the above proof of Theorem 26 for girth $s>6$ only uses $s \neq$ 6 to insure that for a cycle $C$ of length $s$, the graph $K$ obtained from $C$ with its whiskers $1^{\prime}, 4^{\prime}, 6^{\prime}, \ldots, s^{\prime}$ has no edges between the whiskers $1^{\prime}$ and $4^{\prime}$. The rest of the proof only requires $s>5$. In a cycle $C$ of length 6 , any pair of "antipodal" vertices (two vertices of distance 3 from each other in $C$ ) can play the role of 1 and 4 . Therefore, if we assume that there is a pair $i, j$ of antipodal vertices in $C$, such that there is no edge between their whiskers $i^{\prime}$ and $j^{\prime}$, then we can use the same arguments as above to show that $D(G) \leqslant 2$ by labeling $i=1$ and $j=4$. So, we only have to study the case, where there is an edge between all whiskers of antipodal vertices in any 6-cycle in $G$.

Let $C$ be the cycle 123456, where each vertex $i \in\{1, \ldots, 6\}$ has a whisker $i^{\prime}$, and there is an edge between $1^{\prime}$ and $4^{\prime}, 2^{\prime}$ and $5^{\prime}$, and between $3^{\prime}$ and $6^{\prime}$; see the middle part of Figure 19. In particular, those are the only edges between two whiskers, because any other such edge would create a cycle of length strictly less than 6 . Now, if we look at the cycle $1^{\prime} 12344^{\prime}$, with respective whiskers $7,6,2^{\prime}, 3^{\prime}, 5$ and 8 , see the left graph of Figure 18, then there is an edge between its whiskers 5 and 6 . Moreover, there have to be the edges $3^{\prime} 7$ and $2^{\prime} 8$ by assumption. The same holds for the 6 -cycle $1^{\prime} 16544^{\prime}$, compare the right graph of Figure 18, where the edges $5^{\prime} 7$ and $6^{\prime} 8$ have to exist.
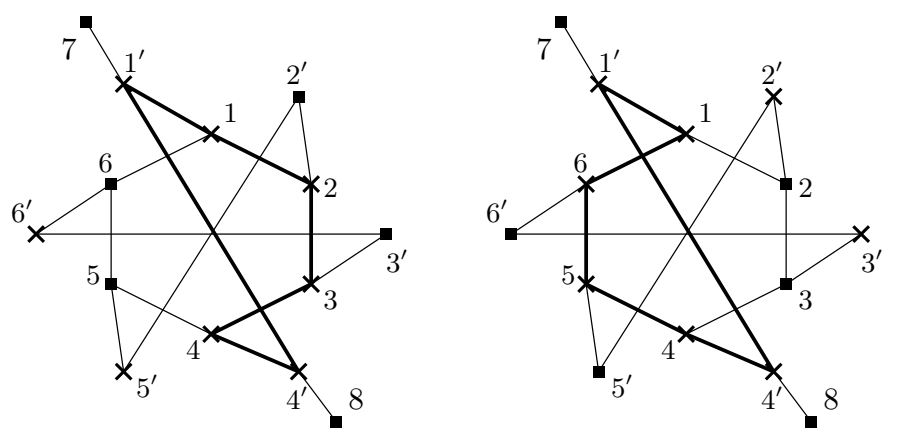

Figure 18: 6-cycles highlighted in $C$ as described in the proof of Theorem 27. Their whiskers are marked by a square.

At this point all fourteen of the vertices have valence 3, so we have the entire graph, see Figure 19. This is the Heawood graph (the dual of the triangulation of the torus with underlying graph $K_{7}$ ).

Now consider the following 2-coloring of the graph. Let 1, 2, 3, 4, 5, 6, 1', 2', 7 be black and the remaining vertices white (see the right graph in Figure 19 for the coloring). In the graph $H$ spanned by the black vertices, 7 is the only vertex of valence 1 adjacent to a vertex of valence 2 (namely $1^{\prime}$ ). Thus, any color-preserving automorphism $\varphi$ fixes 7 . Thus, $\varphi$ also fixes $1^{\prime}$ and hence, also 1 . Since $2^{\prime}$ is the only remaining vertex of valence one, $\varphi$ also fixes 2. Thus, $\varphi$ fixes the remaining vertices of the cycle $C$, so $\varphi$ fixes all black vertices. But then $\varphi$ also fixes the white vertices adjacent to $3,4,5,6$. That leaves only 8 so it must be fixed as well, making $\varphi$ the identity. 

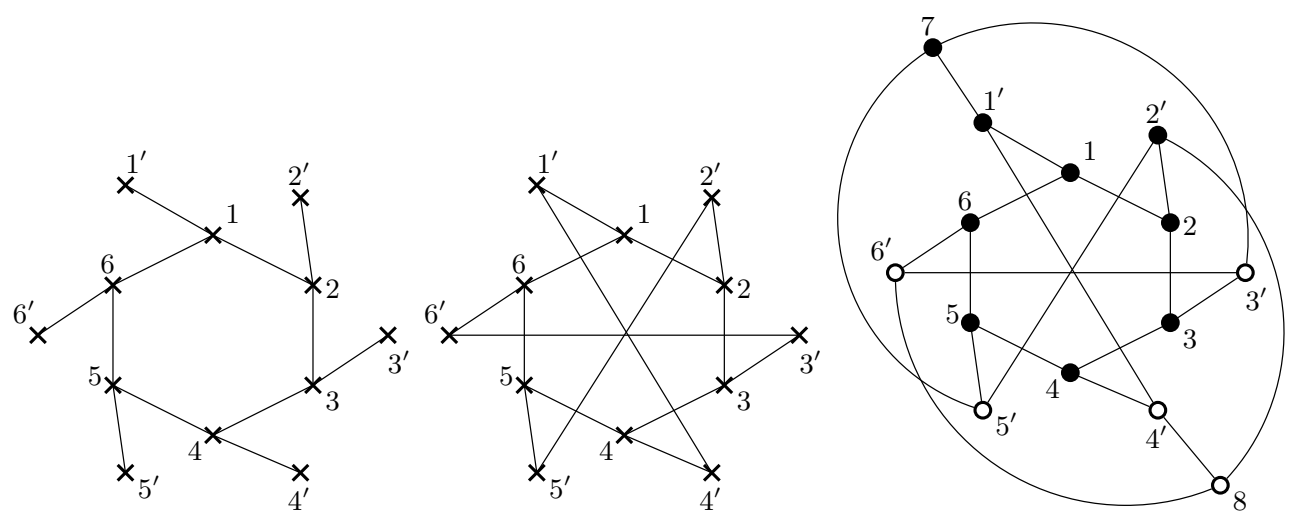

Figure 19: Construction of the graph described in the proof of Theorem 27.

\section{Questions}

There are several questions worth further study. As before, we will denote as $d$ the maximal valence $\Delta(G)$ of a graph $G$.

Higher Valence Can we classify graphs $G$ with $d=D(G)$ ?

As we have observed, if $G=T(n, d)$, then $\Delta(G)=d=D(G)$. We could add edges within each sibling family of size $d-1$ to form a graph $R^{0}(n, d)$ analogous to $R_{n}^{0}$ (the vertices of a sibling family then have valence $d-1)$. We can also attach $d-1$ independent vertices to a sibling family using $K_{d-1, d-1}$ to obtain a graph analogous to $R_{n}^{1}$. There are no analogues for $R_{n}^{2}$ and $R_{n}^{3}$.

We can define a canonical $d-1$ coloring rooted at a graph $K$ such that the only color-preserving automorphism of $G$ fixing the vertices of $K$ is the identity. Then, we have to identify properties of such colorings that restrict the structure of $K$ and $S_{1}(K)$. Note that a variation of the canonical coloring using $d+1$ colors, with color $d+1$ for a vertex $v$, colors $1,2, \ldots, d$ for the neighbors of $v$, and colors $1,2, \ldots, d-1$ is how one gets $D(G) \leqslant d+1$. To show $D(G)=d+1$ only for $G=K_{d+1}$ or $G=K_{d, d}$, one could use the canonical $d$-colorings rooted at an asymmetric vertex-induced subgraph $K$ with color $d$ used only on $K$.

Highly Symmetric Graphs What can one say about vertex transitive, connected graphs with valence $d>3$ and $D(G)>2$ ?

Lehner and Verret [23] have recently classified all such graphs with valence $d=4$. We had originally asked whether the number is finite as it is for $d=3$, but the answer is no. There is one infinite family of lexicographic products $C_{n}\left[2 K_{1}\right]$ (called wreath graphs) with $D(G)=3$ and five others, each with at most 10 vertices. Interestingly, there are none with $D(G)=4$.

Connectivity What is the relationship between vertex or edge connectivity, valence and distinguishing number?

The examples above with $D(G)=d$ are not 2-connected. What happens if we require, 
say, 3-connectivity? For example, we can build a 3-connected graph $G$, such that $D(G)=$ $d-3, d \geqslant 5$, as follows. Let $P$ be a path of length 2 . Attach each of the three vertices of $P$ to a different vertex of one $K_{d}$. The graph $G$ is then 3 -connected and if we color one extremal vertex of $P$ black and the other two white, then the three vertices in $K_{d}$ attached to $P$ are fixed. Therefore, $D(G)=d-3$. As the connectivity goes up, the distinguishing number seems to go down, with finitely many exceptions like $K_{d+1}$.

Infinite Graphs What happens for infinite $G$ with $d>3$ ?

For infinite graphs, we expect that, if $\Delta(G)=d$, then $D(G)<d$, just as for $d=3$. But there are also interesting questions just for such $G$ with $D(G)=2$. As we observed before in Corollary 8, for finite graphs, large enough motion implies $D(G)=2$. The Infinite Motion Conjecture [28] is that, if $G$ is locally finite and $m(G)=\infty$, then $D(G)=2$. On the other hand, for the case $d=3$, we see there is no need for the hypothesis of infinite motion to get $D(G)=2$, and there are other classes of graphs with $D(G)=2$ that do not depend intrinsically on infinite motion [27]. As we observed, however, it is easy to construct an infinite $d$-valent graph $G$ with $D(G)=d-1$. So for $d>3$, we expect infinite motion to be involved.

Motion For cubic graphs, if the motion $m(G)>2$, then $D(G)=2$ with the exception of $Q$ and $P$. For $d>3$, is it the case that, if $m(G)>d$, then $D(G)=2$ with finitely many exceptions?

One might even ask whether $m(G)>2$ gives $D(G)=2$ with finitely many exceptions, but the family of wreath graphs described in [23] provide a negative answer.

Chromatic Distinguishing Number Suppose all colorings are required to be proper (adjacent vertices get different colors). What happens when $d=3$ ?

Collins and Trenk [8] define the chromatic distinguishing number $\chi_{D}(G)$ to be the least $k$ such that $G$ has a proper $k$-coloring whose only color-preserving automorphism is the identity. They prove that $\chi_{D}(G) \leqslant 2 d$ with equality only for $K_{d, d}$ and $C_{6}$. For $d=3$, there is the possibility of classifying graphs with $D(G)=5$, especially in the case that $G$ is bipartite.

In [15] the chromatic distinguishing number of infinite graphs is investigated. For connected graphs of bounded valence $d$ it is shown that $\chi_{D}(G) \leqslant 2 d-1$, and for infinite subcubic graphs of infinite motion this improves to $\chi_{D}(G) \leqslant 4$.

Edge Distinguishing One can also define the distinguishing index (or edge distinguishing number) $D^{\prime}(G)$ as the least $k$ such that some $k$-coloring of the edges of $G$ is preserved only by the identity. In [20] it is shown that $D^{\prime}(G) \leqslant d$ for finite graphs. For infinite graphs $d$ has to be replaced by the supremum of the valences [6].

What happens with $D^{\prime}(G)$, if $d=3$ ?

Cost When $D(G)=2$, the cost $[4,5]$ is the least number of times the color black is used. When $d=D(G)=2$, what can we say about the cost? For cubic graphs this is treated in [18]. 
The canonical coloring tends to use black as few times as possible for $S_{n}(K), n>0$. How close does this number come to the cost?

\section{Acknowledgements}

We owe an enormous debt of gratitude to the two anonymous reviewers of this paper. Their first reports led to a major revision of the paper, including some entirely new, substantial mathematical arguments. They then had to look at two subsequent revisions, and again they gave us extensive reports. This was way beyond the call of duty. In addition, we also thank a third reviewer of the last revision.

\section{References}

[1] M. O. Albertson and K. L. Collins, Symmetry breaking in graphs, Electron. J. Combin. 3 (1996) \#R18.

[2] L. Babai, Asymmetric trees with two prescribed degrees, Acta Math. Hungar. 29 (1977), 193-200.

[3] R. F. Bailey and P. J. Cameron, Base size, metric dimension and other invariants of groups and graphs, Bull. Lond. Math. Soc. 43 (2011), no. 2, 209-242.

[4] D. Boutin, The cost of 2-distinguishing Cartesian powers, Electron. J. Combin. 20 (2013), \#P1.74.

[5] D. Boutin and W. Imrich, The cost of distinguishing graphs, London Math. Society, Lecture Notes Series 436, Cambridge University Press 2017.

[6] I. Broere and M. Pilśniak, The Distinguishing index of Infinite graphs, Electron. J. Combin. 22(1) (2015), \#P1.78.

[7] P. J. Cameron, P. M. Neumann, and J. Saxl, On groups with no regular orbits on the set of subsets, Arch. Math. (Basel) 43 (1984), no. 4, 295-296.

[8] K. L. Collins and A. N. Trenk, The distinguishing chromatic number, Electron. J. Combin. 13 (2006) \#R16.

[9] M. Conder and T. Tucker, The Motion Lemma and distinguishability two, Ars Mathematica Contemporanea, 4 (2011), 63-72.

[10] D. G. Degiorg and K. Klaus, A dynamic algorithm for line graph recognition, Graphtheoretic concepts in computer science (Aachen, 1995), Lecture Notes in Computer Science, 1017, Springer (1995), Berlin, 37-48.

[11] R. Diestel, Graph theory, 3rd ed., Graduate Texts in Mathematics 173, Springer (2006), Berlin.

[12] P. Erdős and A. Rényi, Asymmetric graphs, Acta Mathematica Hungarica, 14 (1963), 295-315.

[13] H. H. Glover, D Marušič, Hamiltonicity of cubic Cayley graph, J. Eur. Math. Soc. 9 (2007) 775-787. 
[14] J. L. Gross, J. Yellen, P. Zhang, Handbook of graph theory, Discrete Mathematics and its Applications (Boca Raton), CRC Press, Boca Raton, FL (2014).

[15] W. Imrich, R. Kalinowski, M. Pilśniak and M.H. Shekarriz, Bounds for Distinguishing Invariants of Infinite Graphs, Electron. J. Combin. 24 (2017), \#P3.6.

[16] W. Imrich, S. Klavžar and V. Trofimov, Distinguishing infinite graphs, Electron. J. Combin. 14 (2007) \#R36.

[17] W. Imrich, S. M. Smith, T. Tucker, and M. E. Watkins, Infinite motion and 2distinguishability of graphs and groups, J. Algebraic Combin. 41 (2015), 109-122.

[18] W. Imrich, T. Lachmann, T. Tucker and G. Wiegel, The cost of colouring vertex transitive cubic graphs, in preparation.

[19] H. A. Jung, Zu einem Isomorphiesatz von H. Whitney für Graphen, Math. Ann. 164 (1966), 270-271.

[20] R. Kalinowski and M. Pilśniak, Distinguishing graphs by edge-colourings, European J. Combin. 45 (2015), 124-131.

[21] S. Klavžar, T.-L. Wong and X. Zhu, Distinguishing labelings of group action on vector spaces and graphs, J. Algebra 303 (2006) 626-641.

[22] F. Lehner, Distinguishing graphs with intermediate growth, Combinatorica 36 (2016), 333-347.

[23] F. Lehner and G. Verret, Distinguishing number of 4-valent vertex-transitive graphs, Ars Mathematica Contemporanea, to appear.

[24] N. Polat and G. Sabidussi, Asymmetrizing sets in trees, Discrete Math. 95 (1991), 271-289.

[25] A. Russell and R. Sundaram, A note on the asymptotics and computational complexity of graph distinguishability, Electron. J. Combin. 5 (1998), \#R23.

[26] À. Seress, Primitive groups with no regular orbits on the set of subsets, Bull. London Math. Soc. 29 (1997), 697-704.

[27] S. M. Smith, T. Tucker and M. E. Watkins, Distinguishability Of Infinite Groups And Graphs, Electron. J. Combin. 19(2) (2012) \#P27.

[28] T. Tucker, Distinguishing Maps, Electron. J. Combin. 18 (2011) R50. 